\title{
PERCEPÇÕES DOS FAMILIARES SOBRE A CAPACITAÇÃO PARA O MANEJO DA TERAPIA NUTRICIONAL PARENTERAL EM DOMICÍLIO
}

\author{
Felipe Leonardo Rigo \\ Fundação Hospitalar do Estado de Minas Gerais \\ felipeleonardorigo@hotmail.com \\ Caroline Soares Rodrigues \\ Fundação Hospitalar do Estado de Minas Gerais \\ carolrodriguespaes@gmail.com \\ Cassidy Tavares Silva \\ Fundação Hospitalar do Estado de Minas Gerais \\ tavarescassidy@gmail.com \\ Thaís Pereira Lopes de Souza \\ Fundação Hospitalar do Estado de Minas Gerais \\ thaispls@live.com
}

\section{RESUMO}

INTRODUÇÃO: A Terapia Nutricional (TN) tem como objetivos a correção da desnutrição prévia, a prevenção ou atenuação da deficiência calórico-proteica, manutenção do estado metabólico com a administração de líquidos, nutrientes e eletrólitos com diminuição da morbidade. A via parenteral é essencial no tratamento intra-hospitalar de crianças com falência intestinal. Os cuidados relacionados a nutrição parenteral em domić́lio é algo complexo e requer dos familiares treinamento prévio ainda em ambiente hospitalar pela equipe multiprofissional. OBJETIVO: Descrever a percepção dos familiares a cerca da capacitação para o manejo da nutrição parenteral (NP) em domicílio. METODOLOGIA: Estudo qualitativo realizado com familiares de crianças que estavam em uso de nutrição parenteral e internadas entre dezembro de 2020 a maio de 202I. Utilizou-se perguntas norteadoras e as respostas foram submetidas à técnica de análise de conteúdo de Bardin. Estudo aprovado pelo Parecer $n^{\circ} 4.443 .630$ e CAAE 40049I20.I.0000.5II9. RESULTADOS: Foram entrevistados 7 cuidadores familiares, 2 para cada criança. Em um caso específico somente a mãe foi treinada, constituído a amostra por 4 mães, 2 pais e I avó. Destes, seis com ensino fundamental completo, um com ensino médio completo, três mães casadas, uma mulher solteira, todos declararam seguir uma religião. Das entrevistas emergiram três categorias temáticas: Percepção sobre o treinamento, Dificuldades/Facilidades e Cuidados em domicílio. Todos os cuidadores avaliaram positivamente o treinamento pelos enfermeiros e afirmaram que a linguagem foi clara e objetiva além de consideraram satisfatório o tempo do treinamento que variou entre I4 a 30 dias. A maioria relatou como principais dificuldades iniciais a troca do curativo do acesso venoso central (CVC) a heparinização do cateter, a manipulação para o preparo da NP, o preenchimento do equipo e conexão da NP ao cateter. Todos os entrevistados concordaram que se sentiram seguros para assumir os cuidados em domicílio após término do treinamento. CONCLUSÃO: O treinamento dos cuidadores é fundamental para os cuidados em domicílio pois, reduz reinternações hospitalares, morbimortalidade e propicia qualidade de vida da criança e família.

PALAVRAS-CHAVE: Nutrição Parenteral. Capacitação. Educação em Saúde 